\title{
Resonantly pumped optical pumping injection cavity lasers
}

\author{
T. C. McAlpine, K. R. Greene, M. R. Santilli, and L. J. Olafsen ${ }^{a)}$ \\ Department of Physics and Astronomy, University of Kansas, Lawrence, Kansas 66045
}

W. W. Bewley, C. L. Felix, I. Vurgaftman, and J. R. Meyer

Code 5613, Naval Research Laboratory, Washington, District of Columbia 20375

\author{
H. Lee ${ }^{\text {b) }}$ and R. U. Martinelli \\ Sarnoff Corporation, CN 5300, Princeton, New Jersey 08543-5300
}

(Received 28 June 2004; accepted 3 August 2004)

\begin{abstract}
An optical parametric oscillator is tuned to the resonance wavelength of the etalon in an optical pumping injection cavity (OPIC) laser with a type-II "W" active region, thereby minimizing the threshold pump intensity and maximizing the output slope efficiency. Previous OPIC experiments employed fixed-wavelength sources with only limited tuning available by adjusting the incident angle. Low threshold pump intensities of $330 \mathrm{~W} / \mathrm{cm}^{2}$ at $100 \mathrm{~K}$ and $14 \mathrm{~kW} / \mathrm{cm}^{2}$ at $300 \mathrm{~K}$ (where the output wavelength is $3200 \mathrm{~nm}$ ) were achieved. The energy conversion efficiency is found to decrease by over a factor of 100 when the pump wavelength is tuned from the resonance condition $(1822 \mathrm{~nm}$ at $300 \mathrm{~K})$ to only slightly off resonance (e.g., $1808 \mathrm{~nm})$. (C) 2004 American Institute of Physics. [DOI: 10.1063/1.1801164]
\end{abstract}

\section{INTRODUCTION}

Quantum well (QW) lasers based on the type-II antimonide heterostructure system have made significant progress toward efficient, room-temperature operation in the midinfrared. ${ }^{1}$ While electrical injection ${ }^{2,3}$ ultimately will be ideal, the optically pumped devices have thus far produced much higher powers than their diode counterparts. ${ }^{4-8}$ To ensure the efficient injection of carriers, these optically pumped lasers have employed two main approaches to maximize the absorption of pump photons in the active region. In the integrated absorber (IA) method first introduced for the type-II antimonides by the Massachusetts Institute of Technology Lincoln Laboratory ${ }^{9-11}$ and further developed at the Air Force Research Laboratory, ${ }^{6-8}$ thick GaInAsSb layers that absorb at $\lambda_{\text {pump }}=1800 \mathrm{~nm}$ surround each active type-II "W" period. The photons absorbed in these layers quickly diffuse to the active QWs.

Another successful approach has been the optical pumping injection cavity (OPIC) laser, ${ }^{12,13}$ in which the active region is enclosed between two $\mathrm{GaSb} / \mathrm{AlAsSb}$-distributed Bragg reflector (DBR) mirrors that induce multiple passes of the pump beam. A schematic is shown in Fig. 1. Tuning the etalon resonance to the pump wavelength (but not to the mid-IR emission wavelength because vertical-cavity lasing is not desired) substantially enhances the net pump absorption efficiency despite a weak absorption for each single pass. The threshold pump intensity is simultaneously reduced, since each QW has multiple opportunities to absorb a given pump photon. The OPIC suppresses internal losses as well, because fewer active QWs are needed to efficiently absorb the pump. This results in less free-carrier absorption of the mid-IR lasing beam by photo-injected carriers. ${ }^{7,13}$ It should

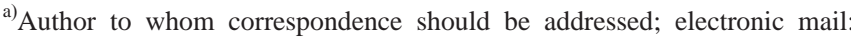
lolafsen@ku.edu

${ }^{b)}$ Present address: Applied Optoelectronics, Inc., Sugar Land, Texas 77478.
}

be further noted with a view toward future work that if the barriers between the QW periods are replaced by GaInAsSb absorbing layers, the hybrid OPIC/IA geometry would combine the advantages of both and allow a further reduction in the number of active QWs.

The first OPIC lasers to be investigated ${ }^{12,13}$ were designed for pumping by a Q-switched Ho:YAG laser emitting at $2100 \mathrm{~nm}$. Whereas the cavity resonance wavelength $\left(\lambda_{\text {cav }}\right)$ for normal incidence, as determined from the transmittance spectrum, occurred at a slightly longer wavelength (e.g.,

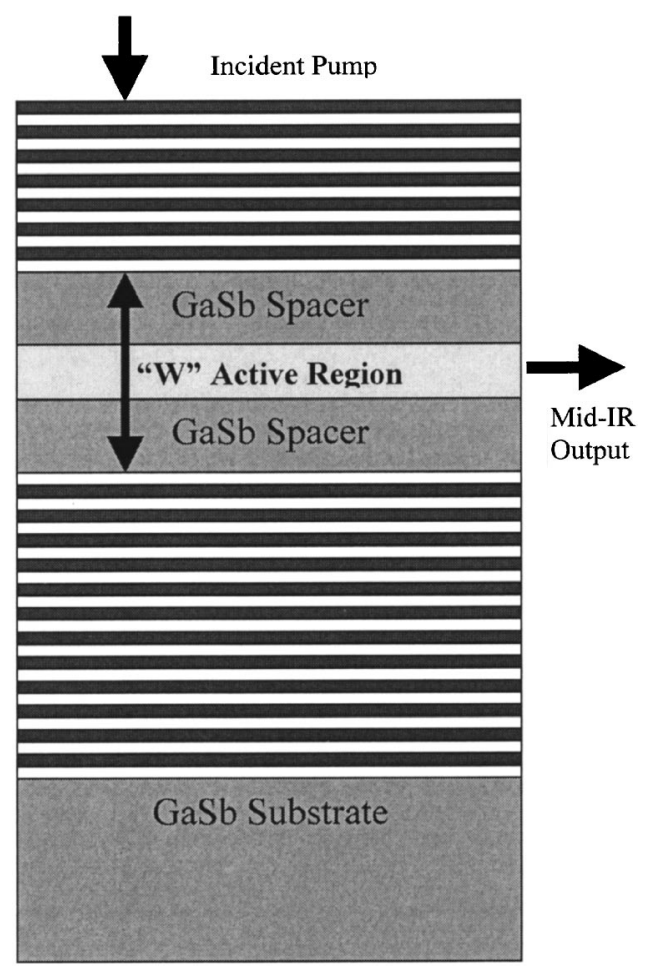

FIG. 1. Schematic of the OPIC laser. 


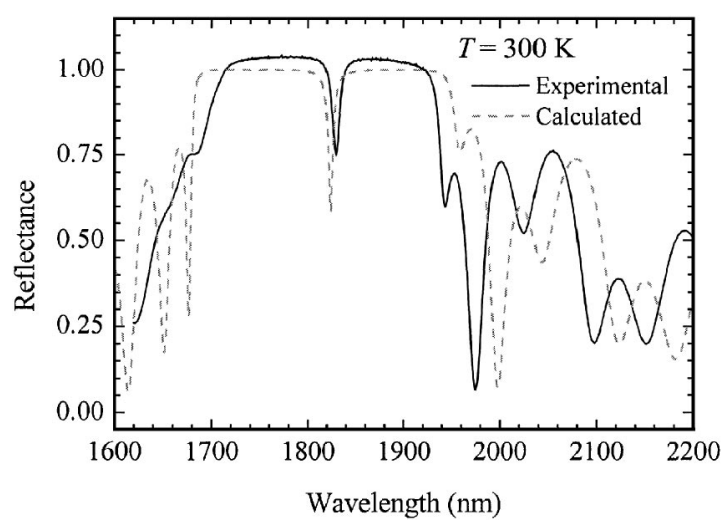

FIG. 2. FTIR reflectance spectrum (solid) for the OPIC structure at normal incidence. The dashed curve represents a model whose only parameter was to adjust all layer thicknesses by the same factor so as to approximately align the resonance wavelength with the experimental value.

$\lambda_{\text {cav }}=2230 \mathrm{~nm}$ for the structure in Ref. 12 at $300 \mathrm{~K}$ ), it was nonetheless possible to reach the resonance condition by angle tuning the pump beam. This approach is viable when $\lambda_{\text {cav }}>\lambda_{\text {pump }}$ and the difference does not exceed $\approx 10 \%$. However, the angle tuning becomes inapplicable whenever $\lambda_{\text {cav }}<\lambda_{\text {pump }}$, besides which, divergence of the angled pump tends to degrade the output beam quality. Much more useful and convenient, at least for research and device-optimization purposes, is to employ a variable-wavelength pump beam. In the present work, an optical parametric oscillator (OPO) is used to study in detail the performance characteristics of a mid-IR OPIC laser with a particularly narrow etalon resonance as a function of $\lambda_{\text {pump }}$.

\section{EXPERIMENT}

The OPIC sample was grown by molecular beam epitaxy on an $n$-GaSb substrate. The strain-compensated type-II W active region (10 periods of $21 \AA \mathrm{InAs} / 34 \AA \mathrm{GaSb} /$ $21 \AA$ InAs/40 $\AA \mathrm{AlSb}$ ) was surrounded on both sides by $100 \AA \mathrm{AlAs}_{0.08} \mathrm{Sb}_{0.92}$ hole-blocking layers and $5213 \AA$ GaSb spacer layers. The top (10 period) and bottom (18.5 period) DBRs contained alternating layers of $1758 \AA \mathrm{AlAs}_{0.08} \mathrm{Sb}_{0.92}$ and $1451 \AA \mathrm{GaSb}$.

Figure 2 illustrates the normal-incidence reflectance spectrum as measured by a Fourier transform infrared (FTIR) spectrometer (solid curve). Note that the apparent maximum reflectivity in the plateau region exceeds unity slightly $(\approx 1.04)$, probably because the reflection of the reference beam was only $\approx 96 \%$. For comparison, the dashed curve shows the results of a simulation, whose only parameter is to adjust all the layer thicknesses by the same constant factor so as to bring the resonance wavelength (indicated by the notch in the plateau) into approximate agreement with the experimental $\lambda_{\text {cav }}$. The theory shows more structure on the shortwavelength side of the plateau because the onset of absorption within the GaSb layers is not included. While this structure was designed for excitation by a quasi-cw diode laser array with $\lambda_{\text {pump }}=1850 \mathrm{~nm}$ at $300 \mathrm{~K}$, the cavity resonance occurs at $1790-1830 \mathrm{~nm}$ (varying slightly with the position on the wafer). Thus, the angle tuning could never bring the OPIC into a resonance with the diode array wave- length, whereas the OPO source at normal incidence easily tunes to $\lambda_{\text {cav }}$ for any temperature of interest. Because the reflectance and lasing samples were taken from different portions of the wafer, the value of $\lambda_{\text {cav }}=1830 \mathrm{~nm}$, indicated by the spectrum in Fig. 2 , is $\approx 9 \mathrm{~nm}$ longer than that determined from the lasing experiments discussed in the succeeding paragraphs. We also point out that even at resonance, the reflectance never falls below $75 \%$. This limits the maximum energy conversion efficiency because it places an upper bound of $25 \%$ on the fraction of the pump beam absorbed within the active quantum wells. More optimized designs can yield absorbed fractions exceeding $80 \%$.

The Spectra-Physics Quanta-Ray MOPO-PO produces wavelengths tunable from the visible to the slightly beyond $2 \mu \mathrm{m}$ when its BBO crystal is pumped at $10 \mathrm{~Hz}$ by the third harmonic (355 nm) from a Spectra-Physics Quanta-Ray Pro$250 \mathrm{Nd}$ :YAG laser. The OPO idler output pulses vary in length from 3.85 to $3.42 \mathrm{~ns}$ as the pump wavelength is increased from 1770 to $1840 \mathrm{~nm}$. The wavelengths of $1770-1840 \mathrm{~nm}$ span the resonances over the full temperature range and hence are of greatest interest in this work. Because both the pulse length of the pump laser and the radiative lifetime in the mid-IR laser are much shorter than the detector response time, the detector functions as an energy integrator in this experiment. For that reason, we will report energy conversion efficiencies rather than power conversion efficiencies as in most previous work on optically pumped mid-IR lasers (for which the pulse length was generally longer than the detector response time). The intensity of the pump beam was controlled by rotating a polarizing cube and was measured with a LaserProbe ratiometer. The idler beam was shaped using cylindrical lenses to control its length and width, which for these experiments resulted in a pump stripe width of $180 \mu \mathrm{m}$ (full width at half maximum). The sample was cleaved to a cavity length of $1.22 \mathrm{~mm}$ and mounted with uncoated facets to the cold finger of an Advanced Research Systems Helitran cryostat using GE varnish.

The output power was measured using a Judson liquidnitrogen-cooled $\mathrm{HgCdTe}$ detector. The peak output power as a function of the peak pump power was measured at and near the resonance condition $\left(\lambda_{\text {pump }}=\lambda_{\text {cav }}\right)$ for temperature ranging from 77 to $350 \mathrm{~K}$, the maximum heater temperature. Spectra were measured at each temperature utilizing an Instruments S.A. TRIAX 550 triple-grating, dual-axis spectrometer. The emission from the OPIC laser was at wavelengths ranging from $2.9 \mu \mathrm{m}$ at $77 \mathrm{~K}$ to $3.2 \mu \mathrm{m}$ at $350 \mathrm{~K}$.

\section{DISCUSSION}

Output energy per pulse versus pump energy density per pulse for a range of pump wavelengths is plotted in Fig. 3. For the shorter-wavelength side of the resonance only, lightlight curves are shown for $T=100$ and $300 \mathrm{~K}$. Longer wavelengths on the far side of the resonance are omitted for clarity because they display very similar behavior. The plots confirm that both the threshold pump energy density (and correspondingly, the threshold pump intensity) and the slope 

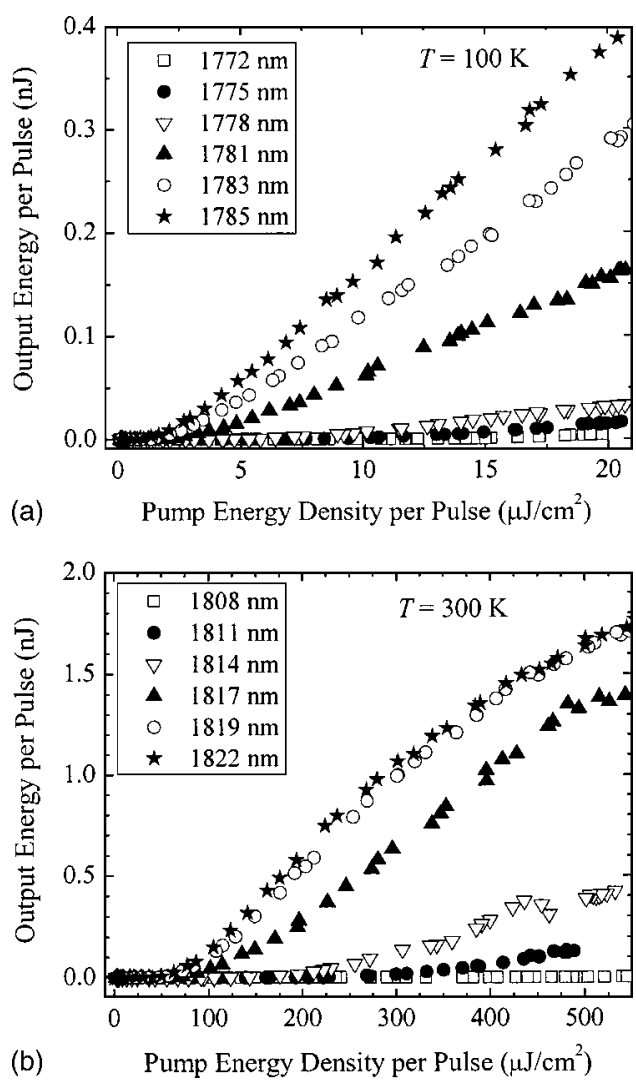

FIG. 3. Output energy per pulse vs pump energy density per pulse for various pump wavelengths at (a) $100 \mathrm{~K}$ and (b) $300 \mathrm{~K}$.

efficiency depend quite strongly on the pump wavelength. The light-light "fans" shown in Fig. 3 are representative of the behavior at all temperatures.

Figure 4 illustrates further that when the pump wavelength is varied, the threshold pump intensity (open points, left axis) has a minimum and the energy conversion efficiency per facet (filled points, right axis) has a maximum at the cavity resonance, with the spectral variations on each side of the resonance being roughly symmetric. For example, when $\lambda_{\text {pump }}$ is tuned to only $13 \mathrm{~nm}$ below the resonance wavelength at $100 \mathrm{~K}$ (i.e., $1772 \mathrm{~nm}$ versus $1785 \mathrm{~nm}$ ), the threshold increases by a factor of 10 and the efficiency decreases by nearly a factor of 30 . At room temperature, the transmission $15 \mathrm{~nm}$ away from resonance (see Fig. 2) is a factor of 12-13 less than at resonance, whereas the efficiency is over 100 times smaller.

There is a strong correlation between the width of the reflectance notch in Fig. 2 and the width of the efficiency peak in Fig. 4(b) because both directly depend on the cavity resonance. The pump absorbance, which dominates the magnitude of the efficiency, is nearly equal to $1-R$ because there is very little transmission when $\lambda \approx \lambda_{\text {cav }}$. This is confirmed by comparing the efficiency spectrum to the plot of $1-R$ (curve) in Fig. 4(b). To obtain the comparison, we first shifted the reflectivity spectrum horizontally by $8.6 \mathrm{~nm}$ to correct for the slight misalignment associated with the variation of $\lambda_{\text {cav }}$ across the wafer. Next, $R(\lambda)$ was divided by a constant factor of $\approx 1.04$ (associated with a small systematic error in the FTIR reference reflectivity), so as to bring its peak value in the plateau region into agreement with the
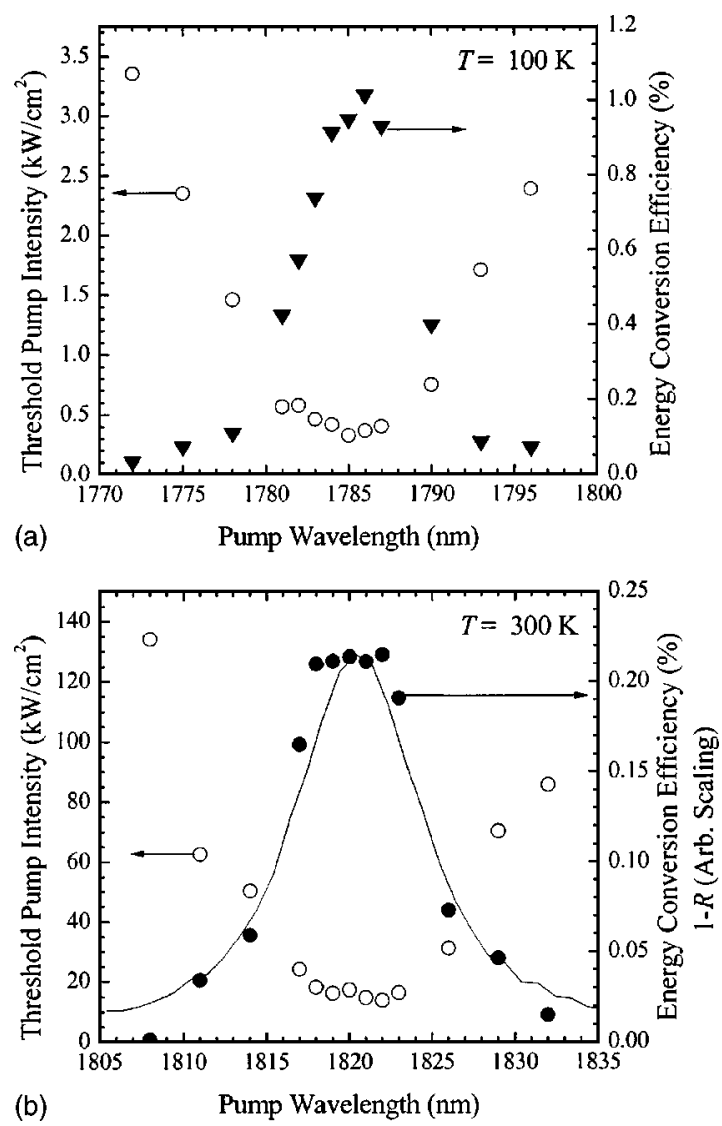

FIG. 4. Threshold intensity (left axis) and energy conversion efficiency per facet (right axis) vs pump wavelength at (a) $100 \mathrm{~K}$ and (b) $300 \mathrm{~K}$, as determined from the light-light curves in Fig. 3. At the OPIC resonance $(\approx 1820 \mathrm{~nm})$, the threshold intensity is minimized and the efficiency is maximized. The curve in (b) is a plot of $1-R$ taken from the reflectivity data in Fig. 2. Here, the experimental reflectivity spectrum was shifted horizontally by $8.6 \mathrm{~nm}$ (to correct for the slight variation of $\lambda_{\text {cav }}$ with position across the wafer), $R$ was normalized to the maximum theoretical value (0.999), and the magnitude of $1-R$ was then arbitrarily rescaled so as to align its maximum with that of the efficiency data.

theoretical value of 0.999 . Finally, the magnitude of $1-R$ was arbitrarily rescaled so as to match its peak height to that of the efficiency data in the figure. We find that the widths of the $1-R$ and efficiency spectra are nearly identical. The more rapid decay in the tail region of the efficiency curve may be associated in part with the difficulty in obtaining an accurate slope when very intense pumping is required because the wavelength is relatively far from resonance (see Fig. 3(b)).

The temperature dependence of the threshold pump intensity at resonance is shown in Fig. 5, where the inset plots $\lambda_{\text {cav }}$ versus temperature. The thresholds of $328 \mathrm{~W} / \mathrm{cm}^{2}$ at $100 \mathrm{~K}$ and $14 \mathrm{~kW} / \mathrm{cm}^{2}$ at $300 \mathrm{~K}$ are lower than those of most conventional optically pumped $\mathrm{W}$ devices. ${ }^{4,14} \mathrm{~W}$-IA lasers have displayed somewhat lower thresholds at low temperatures, ${ }^{7}$ and the W-OPIC lasers from Refs. 12 and 13 had lower $I_{\text {th }}$ at all temperatures up to $300 \mathrm{~K}$ (Ref. 13) because their pump absorbance at resonance was greater.

Energy conversion efficiency per single facet versus temperature is shown in Fig. 6. The efficiencies are determined from the slope of the light-light curves at the corresponding resonant pump wavelength (see inset, Fig. 5). The 


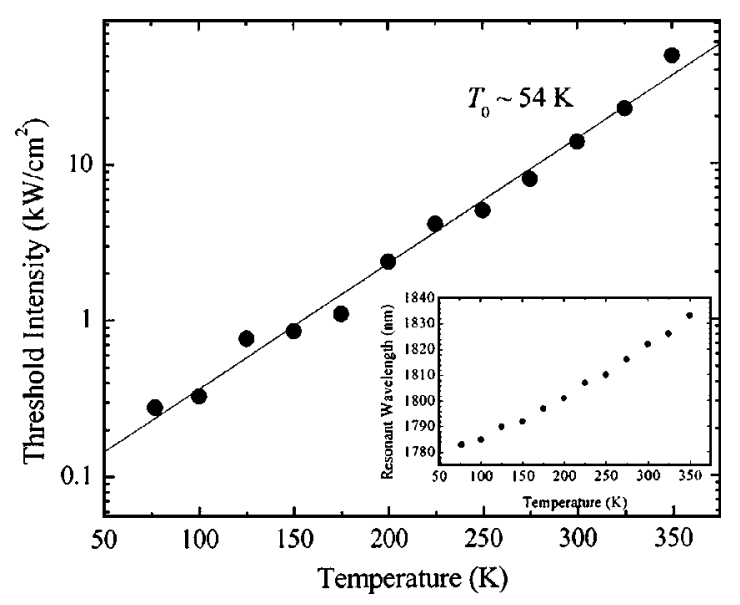

FIG. 5. Threshold intensity vs temperature for resonant optical pumping. Resonant pump wavelengths are plotted vs temperature in the inset. The characteristic temperature is approximately $54 \mathrm{~K}$.

efficiency at $125 \mathrm{~K}$ is $1.25 \%$, and the falloff with temperature is similar to that observed in the earlier OPIC and nonOPIC devices, ${ }^{12}$ reaching approximately $0.22 \%$ at $300 \mathrm{~K}$. The efficiencies at the lowest temperatures are not as high as expected when measured with respect to values above $150 \mathrm{~K}$. At the lowest temperatures, the pump pulse lengths may become shorter than the radiative lifetimes in the OPIC laser.

\section{CONCLUSIONS}

In conclusion, varying the idler wavelength from an OPO pump source made it possible to tune into the OPIC

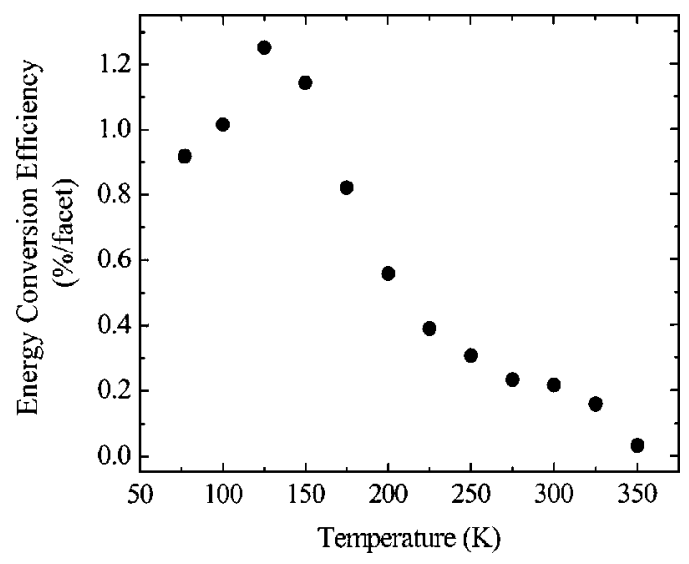

FIG. 6. Energy conversion efficiency per facet vs temperature for the resonantly pumped OPIC. resonance $\left(\lambda_{\text {pump }}=\lambda_{\text {cav }}\right)$ for normal-incidence excitation at any temperature. This greatly facilitates the evaluation of device performance under optimal conditions. As expected, the efficiency displays a sharp maximum and the threshold a distinct minimum when the OPIC laser is pumped at the resonant wavelength for any given temperature. The resonant efficiency exceeds efficiencies only $\sim 13 \mathrm{~nm}$ off resonance by a factor of approximately 30 at $100 \mathrm{~K}$, while thresholds are at least an order of magnitude higher away from the resonant pump wavelength. The spectral width of the efficiency maximum near the resonance was found to match almost exactly that of the peak in $1-R$. Operation of these lasers was achieved to $350 \mathrm{~K}$ and is expected to higher temperatures.

The work at the University of Kansas was supported by the Office of Naval Research (Young Investigator Award), Kansas NSF EPSCoR, and the University of Kansas General Research Fund. The work at NRL was supported by ONR.

${ }^{1}$ I. Vurgaftman, C. L. Felix, W. W. Bewley, D. W. Stokes, R. E. Bartolo, and J. R. Meyer, Philos. Trans. R. Soc. London, Ser. A 359, 489 (2001).

${ }^{2}$ W. W. Bewley et al., Appl. Phys. Lett. 76, 256 (2000).

${ }^{3}$ M. Kim, W. W. Bewley, J. R. Lindle, C. S. Kim, I. Vurgaftman, J. R. Meyer, J. G. Kim, and R. U. Martinelli, Appl. Phys. Lett. 83, 5374 (2003).

${ }^{4}$ W. W. Bewley et al., Appl. Phys. Lett. 73, 3833 (1998).

${ }^{5}$ W. W. Bewley et al., Appl. Phys. Lett. 74, 1075 (1999).

${ }^{6}$ R. Kaspi, A. Ongstad, C. Moeller, G. C. Dente, J. Chavez, M. L. Tilton, and D. Gianardi, Appl. Phys. Lett. 79, 302 (2001).

${ }^{7}$ R. Kaspi, A. Ongstad, G. C. Dente, J. Chavez, M. L. Tilton, and D. Gianardi, Appl. Phys. Lett. 81, 406 (2002).

${ }^{8}$ A. P. Ongstad, R. Kaspi, C. E. Moeller, M. L. Tilton, J. R. Chavez, and G. C. Dente, J. Appl. Phys. 94, 1619 (2004).

${ }^{9}$ A. K. Goyal, G. W. Turner, H. K. Choi, P. J. Foti, M. J. Manfra, T. Y. Fan, and A. Sanchez, IEEE Lasers and Electro-Optics Society Conference Proceedings (LEOS) Annual Meeting, Puerto Rico, 2000), p. 249.

${ }^{10}$ H. K. Choi, A. K. Goyal, S. C. Buchter, G. W. Turner, M. J. Manfra, and S. D. Calawa, Conference on Lasers and Electro-Optics (CLEO 2000), (San Francisco, California, 2000), Vol. 39.

${ }^{11}$ A. K. Goyal, G. W. Turner, A. Sanchez, M. J. Manfra, P. J. Foti, and P. O'Brien, 17th Annual Solid State and Diode Laser Technology Review (Albuquerque, NM, 2004).

${ }^{12}$ C. L. Felix, W. W. Bewley, I. Vurgaftman, L. J. Olafsen, D. W. Stokes, J. R. Meyer, and M. J. Yang, Appl. Phys. Lett. 75, 2876 (1999).

${ }^{13}$ W. W. Bewley, C. L. Felix, I. Vurgaftman, D. W. Stokes, J. R. Meyer, H. Lee, and R. U. Martinelli, IEEE Photonics Technol. Lett. 12, 477 (2000).

${ }^{14}$ C. L. Canedy, W. W. Bewley, C. S. Kim, M. Kim, I. Vurgaftman, and J. R. Meyer, J. Appl. Phys. 94, 1347 (2003). 